\title{
EFEITOS DA PUNIÇÃO DE UMA CLASSE DE RESPOSTAS USANDO DIFERENTES DIMENSÓES E INTENSIDADES DO JATO DE AR QUENTE
}

\section{EFFECTS OF PUNISHMENT OF A RESPONSE CLASS USING DIFFERENT INTENSITIES AND DIMENSIONS OF THE HOT AIR BLAST}

\author{
Bernardo Dutra Rodrigues ${ }^{1}$, Gabriela Souza do Nascimento $^{1}$, \\ Lúcia Cristina Cavalcante ${ }^{2} \&$ Marcus Bentes de Carvalho Neto ${ }^{1}$
}

\author{
${ }^{1}$ UNIVERSIDADE FEDERAL DO PARÁ E ²UNIVERSIDADE DA AMAZÔNIA, BRASIL
}

\begin{abstract}
RESUMO
O Jato de Ar Quente (JAQ) foi utilizado com sucesso como um estímulo punitivo do responder. Contudo, JAQ é um estímulo composto com múltiplas dimensóes potencialmente aversivas (o som, a pressão do ar, o calor produzido diretamente pelo estímulo, e o calor produzido no ambiente experimental) e não se sabe quais delas têm a função de punidor. Além disso, estudos anteriores usaram uma única intensidade do estímulo. $\mathrm{O}$ presente estudo teve dois objetivos principais: identificar que propriedades do JAQ funcionariam como punitivas (Experimento 1) e em que intensidades ele seria efetivo como punidor (Experimento 2). No Experimento 1, nove ratos divididos em três grupos foram expostos a diferentes dimensóes do JAQ: Grupo Som; Grupo Som+Pressão e Grupo Som+Pressão+Aquecimento. Os resultados mostraram que ocorreu supressáo do responder operante somente quando a dimensão Aquecimento foi somada ao Som+Pressão. No Experimento 2, nove ratos, divididos em três grupos, foram expostos a três diferentes intensidades do JAQ: Intensidade Nula; Intensidade Mínima e Intensidade Máxima. Apenas a intensidade máxima foi capaz de promover consistentemente altos níveis de supressão da resposta.
\end{abstract}

Palavras-chave: Jato de Ar Quente, controle aversivo, punição, ratos.

\begin{abstract}
The Hot Air Blast (HAB) procedure has been used successfully as a punishing event. However, HAB is a compound stimulus with multiple potentially aversive dimensions (sound, air pressure, heat generated directly by the stimulus, and the heat produced in the experimental environment) and it is not yet known which is critical as a punisher. Furthermore, previous studies used a single intensity of stimulus. The present study had two main objectives: to identify the properties of HAB that function as a punisher (Experiment 1 ) and intensities at which it would be effective (Experiment 2). In Experiment 1, nine rats, divided into three groups, were exposed to different dimensions of HAB: Sound Group, Sound + Pressure Group and Sound + Pressure + Hot Group. The results indicated that the suppression of operant responding occurred only when the dimension Hot was included with Sound + Pressure. In Experiment 2, nine rats, divided into three groups, were exposed to three different intensities of the composite HAB stimulus: Null Intensity Group, Minimum Intensity Group and Maximum Intensity Group. Only the maximum intensity produced consistent high levels of response suppression.
\end{abstract}

Key words: Hot Air Blast, aversive control, punishment, rats.

Os estudos na área de controle aversivo utilizaram majoritariamente o choque elétrico como estímulo punitivo ou como reforçador negativo em situações de fuga e esquiva (Azrin
\& Holz, 1966/1975, p. 463; Baron, 1991, p. 176; Domjan \& Burkhard, 1993, p. 283). Apesar da sua eficácia comportamental e da sua facilidade e precisão no uso experimental, o choque altera

Agradecimentos especiais a Rafael Teixeira da Silva pela grande contribuição na coleta e discussão dos dados, a Paulo David pelo cuidado com os sujeitos experimentais, à Universidade da Amazônia pelo incentivo ao processo de iniciação científica no curso de Psicologia e aos pareceristas anônimos pelas valiosas sugestões e críticas. Endereço para correspondência: Tv. Castelo Branco, 1170, ap. 205, bloco B. Belém-PA. CEP 66063-000. E-mail: bdr367@yahoo.com.br. 
o organismo de um modo muito específico (Catania, 1998/1999). A generalidade dos efeitos observados sobre o responder, especialmente operante, precisaria ser testada com a adoção sistemática de outros eventos aversivos (Carvalho Neto et al, 2005; Carvalho Neto, Maestri \& Menezes, 2007).

Recentemente, o jato de ar quente (JAQ) vem sendo apresentado como uma alternativa ao choque elétrico em estudos de laboratório com ratos, especialmente como um estímulo punitivo (Carvalho Neto et al, 2005; Carvalho Neto, Maestri \& Menezes, 2007). Nos dois estudos citados, a resposta de pressão a barra (RPB) foi fortalecida previamente sob esquema contínuo de reforçamento (CRF) positivo e tal esquema foi mantido em vigor também durante a fase de punição. Sobreposto ao esquema de reforçamento positivo, um JAQ era apresentado contingente à RPB em dois esquemas diferentes para cada grupo: Punição Contínua e Punição Intermitente (FR3). Os níveis de supressão obtidos no primeiro estudo (Carvalho Neto et al, 2005), após duas sessões de punição, foram de 98,4\% (CRF) e 71,1\% (FR3). No segundo estudo (Carvalho Neto, Maestri \& Menezes, 2007), dois experimentos foram realizados. No Experimento1, após 10 sessões de punição, houve uma supressão de 86,2\% (CRF) e 50,2\% (FR3). No Experimento 2, após 20 sessões de exposição ao mesmo estímulo, índices similares de supressão foram registrados: 92,2\% (CRF) e $57,9 \%$ (FR3). Tais estudos sustentam que o JAQ possui uma função aversiva supressora do responder (punição positiva) e que tal função pode ser mantida mesmo após 20 sessões (Carvalho Neto, Maestri \& Menezes, 2007, Experimento 2).

Contudo, por se tratar de um estímulo composto, com múltiplas dimensões, ainda não se sabe qual (ou quais) delas teria (m) a função supressiva relatada. OJAQ possuiria pelo menos quatro propriedades ou dimensões potencialmente punidoras: o ruído produzido pelo equipamen- to (Som), a pressão exercida pelo deslocamento do ar (Vento), o aumento da temperatura produzido diretamente pelo JAQ sobre o corpo do sujeito dentro do raio de ação do estímulo quando acionado (Aquecimento Específico) e, por último, o aumento da temperatura produzido indiretamente no ambiente experimental após sucessivas apresentações do estímulo durante uma sessão experimental. Os acionamentos continuados do JAQ geravam, independentemente deles tocarem ou não o corpo do sujeito durante o funcionamento do equipamento, uma elevação gradual na temperatura da caixa de condicionamento como um todo. Tal elevação da temperatura foi chamada aqui de Aquecimento Geral.

Para cada uma das quatro dimensões é possível localizar na literatura algum arranjo no qual sua função aversiva foi identificada. Algumas modalidades de som se mostraram aversivas tanto com sujeitos humanos (e.g., Azrin, 1958; Herman \& Azrin, 1964), quanto com pombos (Holz \& Azrin, 1962), gatos (Barry Jr. \& Degelman, 1961) e ratos (e.g., Knutson \& Bailey, 1974; Riess, 1970). Rajadas de ar, não aquecidas, foram igualmente testadas com sucesso como aversivas em macacos (Baker \& Ziegelbauer, 1969; Rohles Jr., 1965) e ratos (Clark, Vasilevsky \& Myers, 2003; Myers, Cohn \& Clark, 2005; Ray Jr., 1966; Ray Jr. \& Lenz, 1968). Temperaturas elevadas em contato com a pele também apresentaram função aversiva em ratos (e.g., Vierck Jr, Kline IV \& Wiley, 2004) e humanos (e.g., Ploghaus, Tracey, Clare, Gati, Rawlins \& Matthews, 2000). A mesma aversividade foi demonstrada diante de temperaturas elevadas no ambiente experimental em ratos (e.g., Caputa, Dok \& Kurowicka, 1998; Green, Hart \& Hagen, 1981; Lipton, Avery \& Marotto, 1970), camundongos (Harikai, Sugawara, Tomogane, Mizuno \&'Tashiro, 2004) e crocodilos (Davidson Jr, 1966).

Além do desconhecimento de qual das dimensões do JAQ exerceria a função punidora, uma única intensidade desse estímulo composto foi até 
aqui utilizada (a "máxima" disponível no equipamento). Como a intensidade do estímulo aversivo é uma das variáveis críticas na determinação dos efeitos da punição (Azrin \& Holz, 1966/1975; Catania, 1998/1999; Estes, 1944; Lerman \& Vorndran, 2002), também seria crucial avaliar em que valores este evento passaria a ser aversivo.

O presente estudo teve dois objetivos principais: identificar que propriedades do JAQ funcionariam como supressoras (Experimento 1) e em que intensidades este estímulo composto seria efetivo como punidor positivo (Experimento 2).

\section{EXPERIMENTO 1}

No primeiro estudo foram testados, de forma cumulativa, três das quatro dimensões potencialmente aversivas que compõem o JAQ (Som, Som+Pressão e Som+Pressão+Aquecimento Específico).

\section{MÉTODO}

\section{Sujeitos}

Nove ratos albinos (Rattus norvegicus, Wistar), machos, experimentalmente ingênuos, com aproximadamente três meses de vida no início do experimento, provenientes do Biotério da Universidade da Amazônia, mantidos em gaiolas-viveiro individuais, em privação de 48 horas de água antes de cada sessão e com fornecimento contínuo de comida (ad libitum). Após cada sessão os ratos recebiam água por uma hora nas gaiolas-viveiro. A temperatura do biotério variava de 20 a $22^{\circ} \mathrm{C}$. Foram seguidas as normas da APA de Condução Ética no Cuidado e Uso de Animais.

\section{Equipamentos e Materiais}

Uma caixa de condicionamento operante (modelo FUNBEC) adaptada com uma tela de arame no lugar do teto e uma barra de acrílico ao invés de alumínio. $\mathrm{O}$ secador de cabelos usado para emitir as dimensões do JAQ era da marca REVLON, modelo RV429AB. A operação manual do secador foi realizada com os mesmos parâmetros previamente descritos (Carvalho Neto et al, 2005; Carvalho Neto, Maestri \& Menezes, 2007), ou seja, o equipamento produzia nesse contexto uma elevação na temperatura, após $5 \mathrm{~s}$, entre $1,5^{\circ} \mathrm{C}$ e $2^{\circ} \mathrm{C}$ em um raio de $10 \mathrm{~cm}$ diretamente sobre a barra, a fim de assegurar que este fosse $\mathrm{o}$ aquecimento que chegava ao sujeito, um ruído de aproximadamente $85 \mathrm{db}$ e uma pressão de 216,5 dyn/cm2 (Intensidade 2, Máxima disponível). O registro das sessões foi feito manualmente.

\section{Procedimento}

Os sujeitos foram divididos em três grupos: Grupo Som (Grupo S): S1, S2 e S3; Grupo Som+Pressão (Grupo SP): SP1, SP2 e SP3; Grupo Som+Pressão+Aquecimento Específico (Grupo SPC): SPC1, SPC2 e SPC3.

No Grupo S, o secador foi colocado sobre a caixa, mas o duto foi direcionado para cima (o JAQ emitido para fora da caixa) e ligado na intensidade 2. Desse modo, apenas o som era apresentado. No Grupo SP, o secador foi colocado sobre a caixa e o duto direcionado na abertura do teto para a parte interna do equipamento, sobre a barra. O secador era acionado também em sua intensidade 2 de pressão e som, mas sem o aquecimento. Nesse arranjo, então, o sujeito era exposto apenas ao som e a pressão. No Grupo SPC, o secador era disposto como descrito no Grupo SP, mas dessa vez o aquecimento era também acionado (intensidade 2 com aquecimento). Nesse grupo estavam presentes assim três dimensões potencialmente aversivas: som, pressão e Aquecimento Específico, aqui chamado simplesmente de calor.

Os sujeitos foram submetidos a cinco fases: $1^{\mathrm{a}}$ ) Nivel operante da RPB (uma sessão); $2^{\mathrm{a}}$ ) 
Treino ao bebedouro seguido da Modelagem da RPB (uma a três sessões); $3^{\text {a }}$ ) Fortalecimento contínuo (CRF) da RPB (cinco sessões); $4^{\mathrm{a}}$ ) Uso do JAQ com a apresentação contingente a cada emissão da RPB, por cinco segundos, da (s) dimensão (ões) do estímulo avaliada (s) e manutenção do reforçamento com água em CRF (dez sessões), deve-se ressaltar que cada resposta que ocorresse enquanto o JAQ era liberado não prolongava nem iniciava um novo período de punição, mas era reforçada. Para que a resposta fosse punida novamente o sujeito deveria responder após os cinco segundos de duração do JAQ; 5 $5^{\mathrm{a}}$ ) Recondicionamento da $\mathrm{RPB}$ (cinco sessões).

\section{RESULTADOS E DISCUSSÃO}

Os três sujeitos do Grupo S (Som) apresentaram uma redução na freqüência da RPB na primeira sessão em que a dimensão Som foi apresentada (decréscimos de 8\%, S1, 29,4\%, S2, e $37,5 \%$, S3), observou-se, no entanto, que essa queda não foi consistente ao longo das sessões. De fato, houve um aumento na média da freqüência da RPB na fase como um todo (acréscimos de 3,9\%, S1, 10,9\%, S2, e 13,2\%, S3).

$\mathrm{Na}$ fase de Recondicionamento da RPB, os desempenhos dos sujeitos foram bastante variados: S2 e S3 apresentaram acréscimo de freqüência na primeira sessão em relação à última em que ocorreu a apresentação da dimensão Som (S2: 3,9\%; S3: 8,1\%), enquanto S1 apresentou um decréscimo (S1: 26,3\%); S1 e S2 apresentaram um aumento da freqüência de RPB em relação à média da fase de apresentação da dimensão Som (S1: 3,9\%; S2: 12,8\%), enquanto o S3 apresentou um decréscimo (S3: 10,5\%) (ver Figura 1).

Todos os sujeitos do Grupo SP (Som + Pressão) apresentaram uma queda na freqüência da RPB na primeira fase em que as dimensões programadas do JAQ foram apresentadas (decréscimos de 67,5\%, SP1, $78,7 \%$, SP2, e 49,2\%, SP3). Porém, quando se compara a média de toda esta fase com

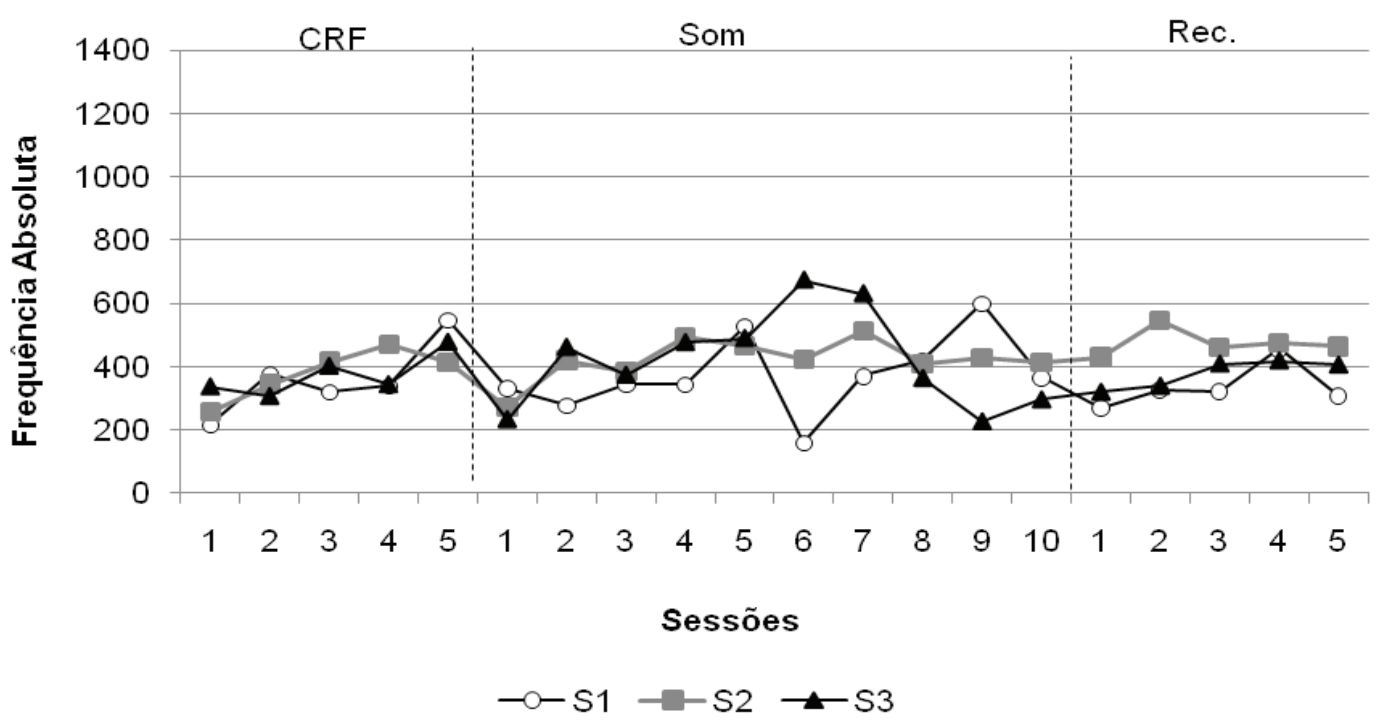

Figura 1. Frequência absoluta da RPB dos sujeitos do Grupo S (Som) nas fases de Fortalecimento (CRF), apresentação da dimensão manipulada do JAQ (Som) e Recondicionamento da RPB (Rec.). 
a média obtida na fase anterior de fortalecimento em CRF, nota-se que o sujeito SP2 teve um desempenho diferenciado dos demais sujeitos. Enquanto os outros sujeitos mantiveram um decréscimo médio de 36,7\% (SP1) e 5,9\% (SP3), o sujeito SP2 apresentou um acréscimo médio de 11,4\%. Já na fase de Recondicionamento todos os sujeitos deste grupo apresentaram um acréscimo em relação à última fase de apresentação das dimensões Som+Pressão (SP1: 12,1\%; SP2: 2,2\%; SP3: 15,8\%). Novamente, quando se e consistente na freqüência da RPB durante toda a fase em que as dimensões Som+Pressão+Calor foram apresentadas. Na primeira sessão de apresentação das dimensões, a supressão inicial, se comparada com a última sessão de CRF, foi de 98,4\% (SPC1), 92,4\% (SPC2) e 98,6\% (SPC3). Tal desempenho foi mantido no decorrer desta fase sendo que em média a supressão geral (se comparada com a média da fase de CRF) foi de 99,1\% (SPC1), 89,6\% (SPC2) e 99,6\% (SPC3). Durante o Recondicionamento, os sujeitos SPC1 e SPC3 apresentaram na primeira sessão uma supressão

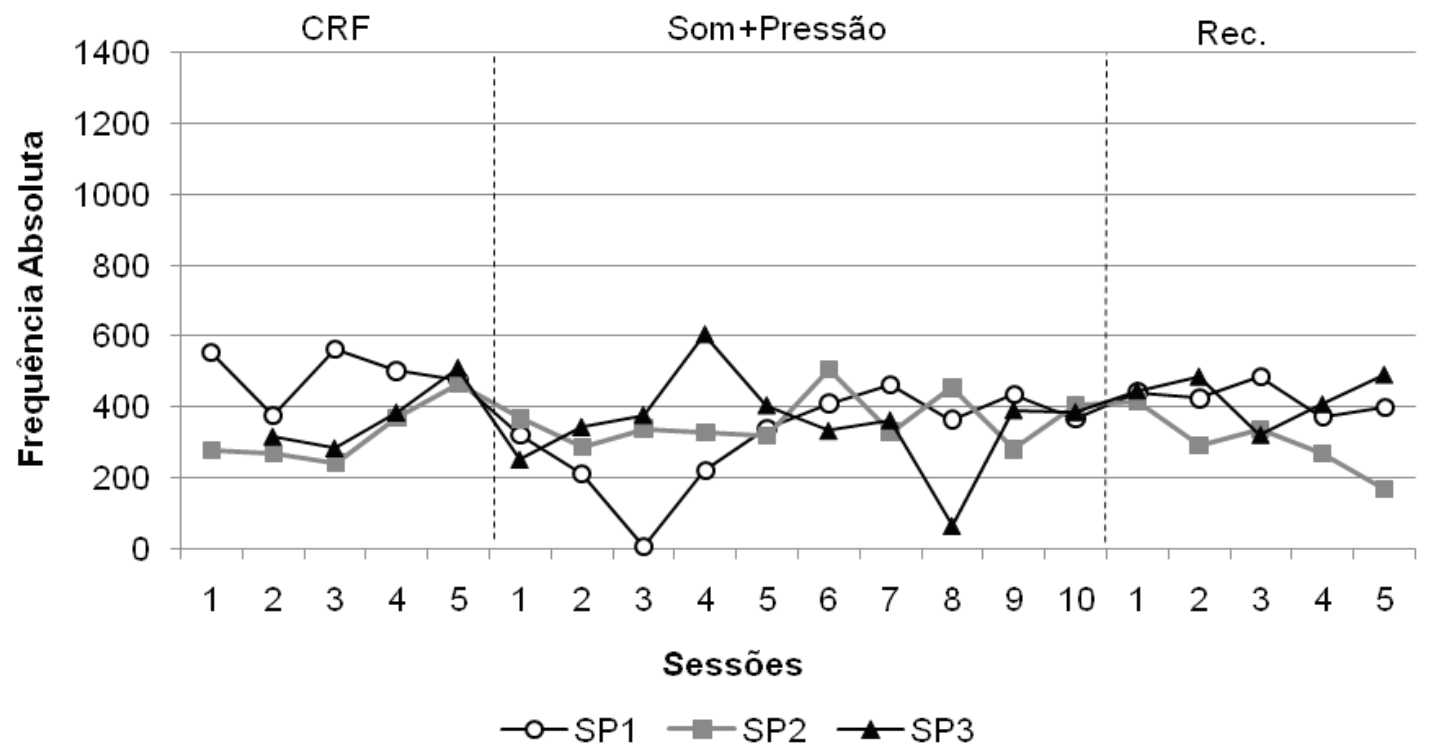

Figura 2. Frequência absoluta da RPB dos sujeitos do Grupo SP (Som + Pressão) nas fases de Fortalecimento (CRF), apresentação da dimensão manipulada do JAQ (Som + Pressão) e Recondicionamento da RPB (Rec.).

analisa a média da fase é possível observar que o sujeito SP2 teve um desempenho diferenciado dos demais. Enquanto os outros sujeitos apresentaram um acréscimo médio de 35,6\% (SP1) e 22,4\% (SP3), o sujeito SP2 apresentou um decréscimo de 18,3\% no decorrer da fase de Recondicionamento (ver Figura 2).

Os sujeitos do Grupo SPC, diferente dos demais grupos, mostraram uma redução acentuada total na freqüência da RPB, sendo que o SPC1 na sessão anterior emitiu apenas uma RPB e o SPC3 apresentou uma supressão total desde a $9^{a}$ sessão em que o JAQ foi apresentado e esta perdurou até a $2^{\mathrm{a}}$ sessão de Recondicionamento. Nas $4^{\mathrm{a}}$ e $5^{\mathrm{a}}$ sessões de Recondicionamento estes dois sujeitos apresentaram um acréscimo abrupto na freqüência da RPB e estas foram superiores as freqüências apresentadas na fase de CRF. O sujeito SPC2 apresentou um acréscimo de $993 \%$ na freqüência 


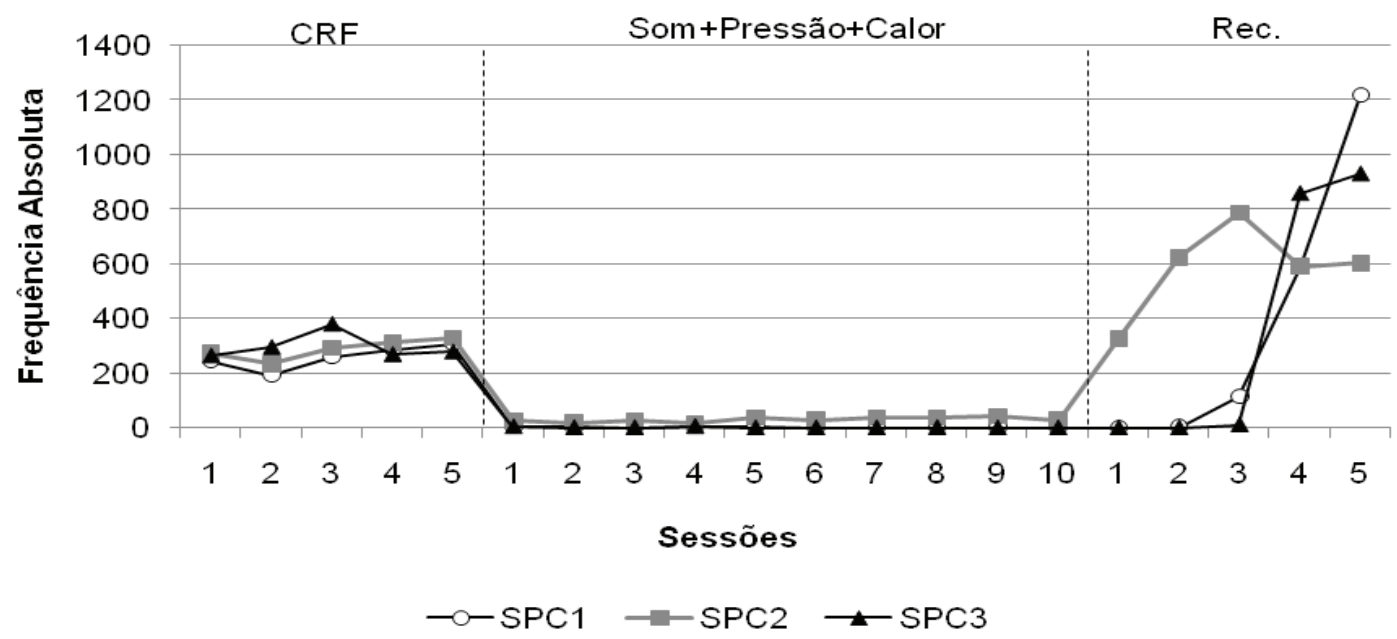

Figura 3. Frequência absoluta da RPB dos sujeitos do Grupo SPC (Som + Pressão + Calor) nas fases de Fortalecimento (CRF), apresentação da dimensão manipulada do JAQ (Som + Pressão + Calor) e Recondicionamento da RPB (Rec.).

da RPB na primeira sessão de Recondicionamento, quando comparado com a última sessão de apresentação das dimensões manipuladas. Destaca-se que a recuperação do responder para este sujeito se deu de forma gradual, diferente dos sujeitos SPC1 e SPC2. Em média, os sujeitos apresentaram um aumento de 1.604,3\% (SPC1), 1.860,7\% (SPC2) e 2.988,3\% (SPC3) da RPB na fase de Recondicionamento, quando comparado com a média da fase anterior (ver Figura 3).

A análise da freqüência da RPB na fase de apresentação da (s) dimensão (ões) nos grupos S, SP e SPC indica que a dimensão Aquecimento Específico, presente apenas no último grupo (SPC), precisa estar presente para produzir a regularidade e os níveis de supressão compatíveis com os registrados nos estudos anteriores usando o JAQ (Carvalho Neto et al, 2005; Carvalho Neto, Maestri \& Menezes, 2007). Nos estudos mencionados e no Grupo SPC foram observadas médias de supressão acima de $85 \%$ e esse efeito foi constante durante as sessões em que o JAQ estava presente. Todavia, não é possível asse- gurar que a dimensão Aquecimento Específico sozinha seja a responsável pela redução na freqüência observada. Como o delineamento era cumulativo, existe a possibilidade de que o resultado final seja um efeito de somação de estímulos e não da introdução da última dimensão manipulada.

No Grupo S foi observada também a supressão da RPB quando da introdução inicial do estímulo (som). Contudo, ela foi parcial e, em geral, restrita à 1 a sessão dessa fase. No Grupo SP também foi registrado um padrão similar de supressão parcial, mas para um deles (SP1) essa supressão foi mantida pelas três sessões iniciais e para outro (SP2) foi mantida nas duas primeiras. Após as primeiras sessões dessa fase, foram observados padrões alternados e irregulares de aumento e queda na freqüência.

No experimento de May, Tolman e Schoenfeldt (1967) sobre supressão condicionada, observou-se que o grupo que não teve uma pré-exposição ao estímulo neutro (som), antes do pareamento, apresentou uma maior supressão do operante, quando comparado 
com o grupo que teve uma experiência prévia com o som. Tais autores sugerem que estes resultados estão diretamente relacionados ao efeito "novidade" do som, que eliciaria respostas de orientação, o que ocasionalmente concorreria com o operante, levando indiretamente a sua supressão. É possível que um efeito similar possa ter acontecido nas sessões iniciais quando da apresentação do som nos Grupos S e SP, contudo os dados como foram coletados não permitem uma análise desse tipo.

Dois dos três sujeitos apresentaram supressão total da RPB da primeira e até a terceira sessão (SPC1 e SPC3, respectivamente) durante o JAQ, indicando que tal evento funcionou como um estímulo punitivo severo, afetando a freqüência da RPB mesmo após a sua retirada. Observouse também nas demais sessões dessa fase um acréscimo significativo na freqüência da RPB, chegando a níveis superiores aos apresentados na fase de CRF. Este padrão é conhecido como efeito de contraste (Azrin \& Holz, 1966/1975; Estes, 1944; Lerman \& Vorndran, 2002). Os dados desse estudo replicam os obtidos previamente com choque elétrico (e.g., Estes, 1944).

\section{EXPERIMENTO 2}

O Experimento 1 indicou que a supressão da RPB foi mais acentuada e consistente no Grupo SPC, no qual estavam presentes as três dimensões manipuladas (som+ pressão+calor). A intensidade do estímulo composto JAQ utilizada nesse e nos estudos anteriores foi sempre a maior disponível no equipamento (Máxima). O objetivo deste experimento foi avaliar se as outras intensidades menores do JAQ Nula e Mínima) também seriam capazes de suprimir o responder.

\section{MÉtodo}

\section{Sujeitos}

Nove ratos albinos com as mesmas características, condições de alojamento e esquema de privação e alimentação descritas no Experimento 1. Novamente foram seguidas as normas da APA de Condução Ética no Cuidado e Uso de Animais.

\section{Equipamentos e materiais}

Os mesmos descritos no Experimento 1.

O modelo de secador de cabelos utilizado no Experimento 1 e nos estudos anteriores (Carvalho Neto et al, 2005; Carvalho Neto, Maestri \& Menezes, 2007) permite três níveis de intensidade: Nula, Mínima e Máxima.

A operação manual do secador nas suas três intensidades produzia diferentes variações de temperatura, pressão e ruído ao final de cinco segundos. A intensidade Nula produzia uma elevação na temperatura de $0^{\circ} \mathrm{C}$ em um raio de $10 \mathrm{~cm}$, um ruído de aproximadamente $80 \mathrm{db}$ e uma pressão de 216,5 dyn/cm2. A intensidade Mínima produzia uma elevação na temperatura de $1^{\circ} \mathrm{C}$ em um raio de $10 \mathrm{~cm}$, um ruído de aproximadamente $75 \mathrm{db}$ e uma pressão de $216,5 \mathrm{dyn} /$ $\mathrm{cm} 2$. A intensidade Máxima, a mesma utilizada no Experimento 1, produzia uma elevação na temperatura entre $1,5^{\circ} \mathrm{C}$ e $2^{\circ} \mathrm{C}$ em um raio de 10 $\mathrm{cm}$, um ruído de aproximadamente $85 \mathrm{db}$ e uma pressão de aproximadamente 216,5 dyn/cm2.

O registro das sessões foi feito como no Experimento 1.

\section{Procedimento}

Os sujeitos foram divididos em três grupos de acordo com as intensidades do JAQ avaliadas: Intensidade Máxima (Grupo MA): MA1, MA2 e MA3; Intensidade Mínima (Grupo MI): MI1, MI2 e MI3 e; Intensidade Nula (Grupo N): N1, N2 e N3. Os sujeitos foram submetidos a cinco fases: $1^{\text {a }}$ ) Nível ope- 
rante (uma sessão); $2^{\mathrm{a}}$ ) Treino ao bebedouro e Modelagem da RPB (uma a três sessões); $3^{a}$ ) Fortalecimento em CRF (quatro sessões); $\left.4^{a}\right)$ Apresentação contingente à RPB do JAQ na intensidade avaliada e manutenção do reforçamento com água (quatro sessões); $5^{\mathrm{a}}$ ) Recondicionamento da RPB (quatro sessões). Todas as sessões tiveram duração de uma hora. As respostas registradas foram as mesmas do Experimento 1.

\section{RESULTADOS \& DISCUSSÃO}

No Grupo N, dois dos três sujeitos apresentaram uma redução na freqüência da RPB na primeira sessão de JAQ, com decréscimo de $36,4 \%$ (N1) e $41 \%$ (N3). O sujeito N2, por sua vez, apresentou um acréscimo de $8,9 \%$. Este mesmo padrão foi observado na média geral de freqüência dessa fase em relação à média da freqüência de CRF, com decréscimo de 16,7\% (N1) e 31,2\% (N3), enquanto que o sujeito N2 obteve um acréscimo de $20,7 \%$. Já na fase de
Recondicionamento, todos os sujeitos apresentaram um acréscimo na freqüência da RPB tanto na primeira sessão desta fase (acréscimo de, NI: $13 \%$; N2: 16,8\%; N3: 34,6\%), quanto na média geral comparada com a média global da fase anterior (acréscimo de, N1: 50,6\%; N2: 8,6\%; N3: 19,9\%) (ver Figura 4).

Apesar da queda inicial para dois dos sujeitos, essa se deu na primeira sessão e nas sessões seguintes observou-se uma recuperação gradual do responder. Portanto, o efeito supressivo não ocorreu com todos os sujeitos (2 de 3) e quando registrado, foi temporário.

No Grupo MI, dois dos sujeitos também apresentaram decréscimos na primeira sessão de JAQ: 28,6\% (MI1) e 78\% (MI2). O outro sujeito deste grupo apresentou uma elevação na freqüência do responder nesta mesma sessão de 44\% (MI3). Ao se comparar a média geral da fase de JAQ com a média de CRF, os dois sujeitos que apresentaram o decréscimo na primeira sessão também apresentaram decréscimos nesse valor

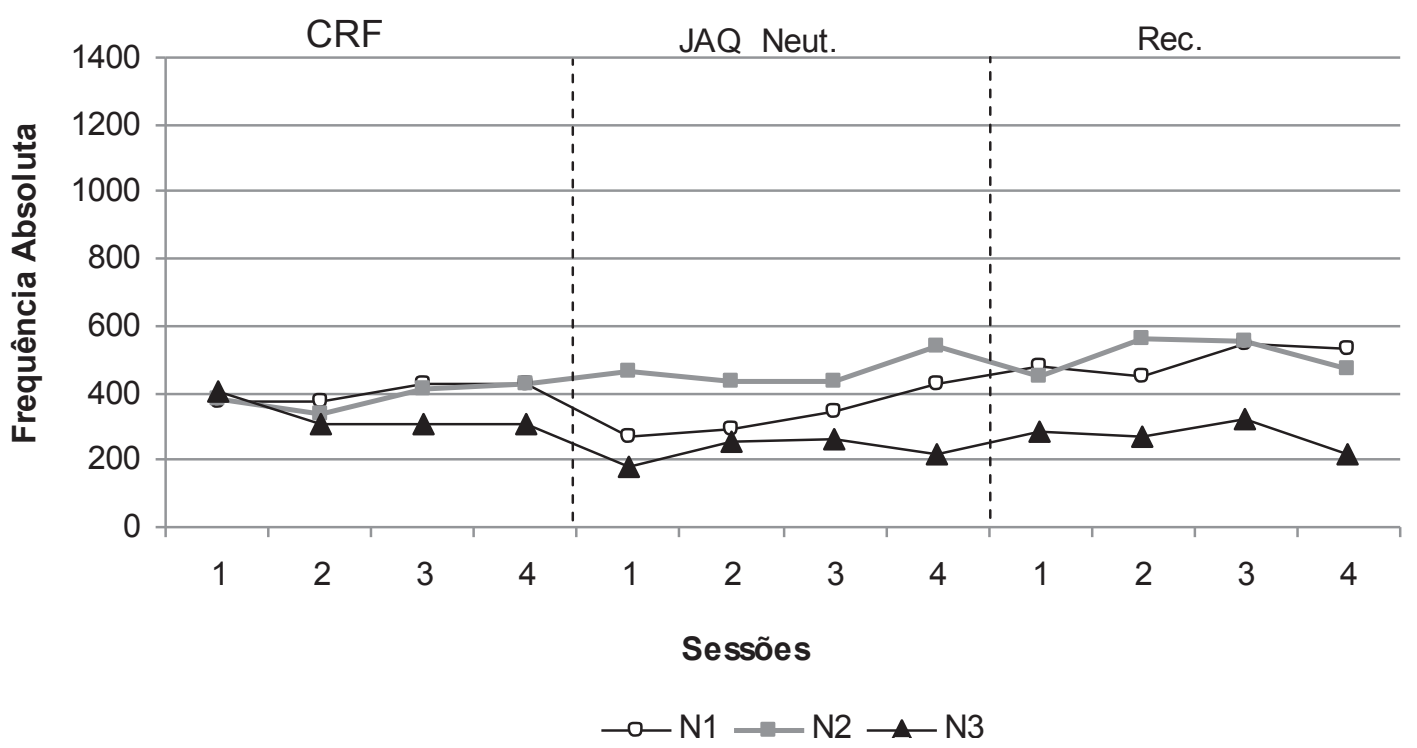

Figura 4. Frequência absoluta da RPB dos sujeitos do Grupo com intensidade 0 (Neutro, N) nas fases de CRF, apresentação do JAQ e Recondicionamento. 


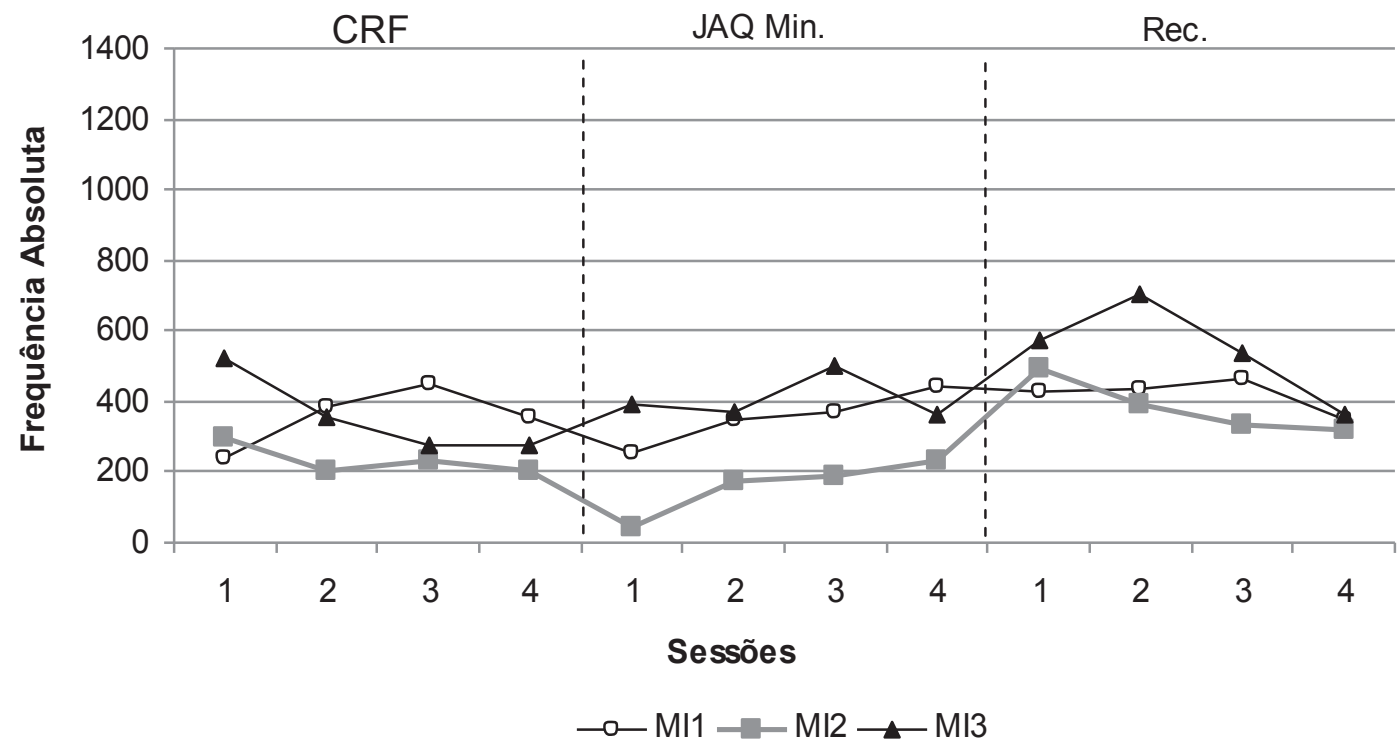

Figura 5. Frequência absoluta da RPB dos sujeitos do Grupo com intensidade 1 (Mínima, MI) nas fases de CRF, apresentação do JAQ e Recondicionamento.

médio: 1,4\% (MI1) e 32,3\% (MI2). MI3, por sua vez, apresentou um acréscimo na freqüência de 14\%. Na primeira sessão da fase de Recondicionamento, novamente, o sujeito MI1 apresentou um desempenho diferenciado dos outros sujeitos deste grupo. Enquanto MI2 e MI3 tiveram uma elevação na freqüência do responder de: 114,4\% (MI2) e 58,9\% (MI3), MI1 apresentou uma redução de 2,5\%. Contudo, comparando-se as médias de cada fase, todos os sujeitos apresentaram um acréscimo na freqüência do responder (MI1: 18,9\%; MI2: 49,5\%; MI3: 33,8\%) (ver Figura 5).

No Grupo MI observou-se um padrão similar ao do Grupo N: queda inicial para dois dos sujeitos, na primeira sessão, e recuperação gradual do responder nas sessões seguintes de JAQ. Novamente o efeito supressivo não ocorreu com todos os sujeitos (dois de três) e quando foi registrado, foi temporário.

A supressão mais acentuada e sistemática do responder ocorreu no Grupo MA, atingindo as médias de 85\% (MA1), 92\% (MA2) e $86,3 \%$ (MA3) (média da fase de CRF X média de JAQ). Na primeira sessão em que o JAQ foi apresentado, as supressões foram de 91,2\% (MA1), 96,4\% (MA2) e 93,2\% (MA3) (última sessão da fase de CRF X primeira de JAQ). Durante o Recondicionamento houve uma recuperação do responder para todos os sujeitos, atingindo a média de 94,1\% (MA1), 62,7\% (M2) e 81,9\% (MA3) (média da fase de JAQ X média de Recondicionamento). Quando se examina a primeira sessão de Recondicionamento, todos também tiveram um acréscimo na freqüência da RPB (MA1: 306,1\%; MA2: 484,6\%; MA3: 495,4\%). O sujeito MA1, devido a falhas técnicas, realizou apenas três sessões de Recondicionamento (ver Figura 6).

Em suma, a análise da freqüência da RPB na fase de JAQ nos grupos N, MI e MA indicou que apenas o último deles, intensidade máxima (2), foi capaz de promover, de modo consistente, níveis de supressão acima de 90\%, replicando os dados obtidos no Grupo SPC do Experimento 1 e dos estudos anteriores com JAQ (Carvalho Neto et al, 2005; Carvalho 


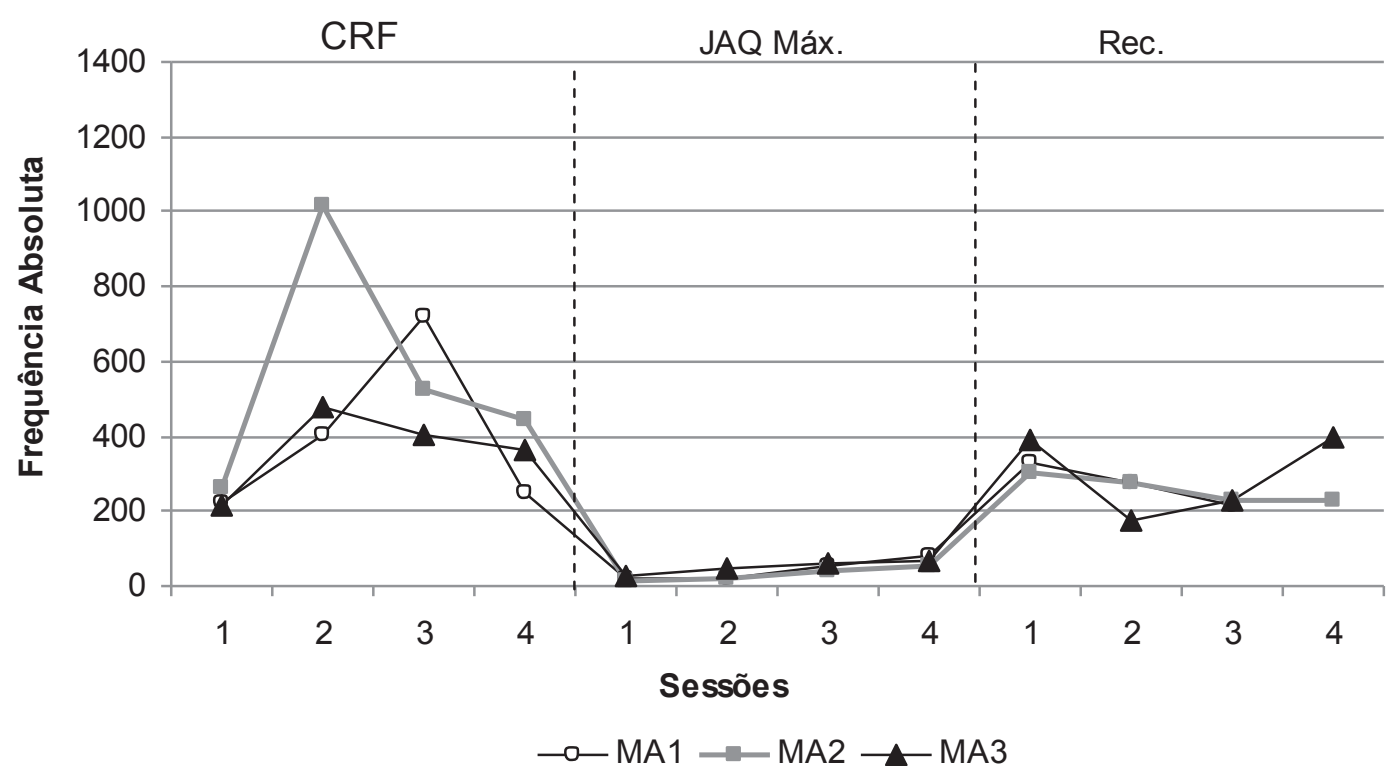

Figura 6. Frequência absoluta da RPB dos sujeitos do Grupo com intensidade 2 (Máxima, MA) nas fases de CRF, apresentação do JAQ e Recondicionamento.

Neto, Maestri \& Menezes, 2007). De acordo com Azrin e Holz (1966/1975), a intensidade do estímulo aversivo é uma variável determinante para a redução da freqüência de uma resposta durante o processo de punição, aspecto confirmado no Experimento 2.

Embora haja semelhanças entre os resultados da fase JAQ nos Grupos SPC do Experimento 1 e MA do Experimento 2 (que usaram a mesma intensidade do estímulo), há diferenças no desempenho na fase de Recondicionamento. Não foi observada uma supressão total do responder nas primeiras sessões do Grupo MA, diferentemente do que ocorreu no Grupo SPC. Um aspecto a ser considerado nessa análise é a diferença do número de sessões de punição nos dois Experimentos (10 no Experimento 1 e 4 no Experimento 2), indicando que esta é, possivelmente, uma outra variável relevante para a produção do efeito de contraste e da manutenção da supressão na ausência do estímulo aversivo.
Apesar da variabilidade intra-sujeito observada no Grupo N, os dados deste grupo foram semelhantes aos do Grupo SP do Experimento 1, tanto na fase de apresentação do estímulo, quanto na fase de Recondicionamento, confirmando que as dimensões Som + Pressão não funcionaram, nas intensidades usadas, como estímulos punitivos.

Apesar de ter sido previamente demonstrada a função aversiva do jato de ar sem aquecimento (e.g., Baker \& Ziegelbauer, 1969; Clark, Vasilevsky \& Myers, 2003; Myers, Cohn \& Clark, 2005; Ray Jr., 1966; Ray Jr. \& Lenz, 1968; Rohles Jr., 1965) e do som sozinho (e.g., Azrin, 1958; Barry Jr. \& Degelman, 1961; Herman \& Azrin, 1964; Knutson \& Bailey, 1974; Riess, 1970), nas intensidades aqui adotadas e no modo como foram apresentados, tais estímulos não se mostraram aversivos intensos. Por exemplo, Myers, Cohn \& Clark (2005) usaram jatos de ar pressurizados emitidos através de quatro tubos de 6,5 $\mathrm{mm}$ de diâmetro dispostos nas laterais de uma shuttlebox. A menor pressão utilizada nesse contexto foi de 8,5 psi, equivalente 
a 586,05 dyn/cm2. A maior utilizada com JAQ, no atual estudo, foi de 216,5 dyn/cm2 . Na literatura citada, os jatos de ar e os sons que funcionam como aversivos são ou mais intensos que os aqui usados ou são empregados de modo diferente. Por exemplo, Belova, Paton, Morrison e Salzman (2007) usaram a técnica de disparar jatos de ar com intensidades menores que as aqui descritas, mas no rosto dos sujeitos. O local de ação do jato não aquecido é, aparentemente, também uma dimensão importante para que ele funcione como aversivo.

Em suma, os dados obtidos no Experimento 1 demonstraram que o Aquecimento Específico precisa estar presente para que se observe uma elevada (acima de 85\%) e consistente (até 10 sessões) supressão do responder. No Experimento 2, por sua vez, demonstrouse que intensidades menores do JAQ não são capazes de produzir quedas acentuadas e regulares no responder.

Das quatro dimensões indicadas como potencialmente aversivas no JAQ, foram testadas três: o ruído produzido pelo equipamento (som), a pressão exercida pelo deslocamento do ar (vento) e o aumento da temperatura produzida diretamente pelo estímulo (Aquecimento Específico). Entretanto, como já mencionado, ainda não seria possível afirmar que esta última dimensão sozinha seja a responsável pela redução na freqüência observada. Como o delineamento era cumulativo, existe a possibilidade de que o resultado final seja um efeito de somação de estímulos e não da introdução da última dimensão manipulada. Tal possibilidade precisaria ser verificada através de um procedimento no qual cada dimensão seria apresentada separadamente.

Além das três dimensões estudadas, restaria ainda testar uma quarta dimensão: o aumento da temperatura produzido no ambiente experimental (Aquecimento Geral). Futuras pesquisas poderão avaliar os efeitos dessa dimensão na supressão do responder.

\section{REFERENNCIAS}

Azrin, N. H. (1958). Some effects of noise on human behavior. Journal of the Experimental Analysis of Behavior, 1, 183-200.

Azrin, N. H., \& Holz, W. C. (1975). Castigo. In W. K. Honig, (Ed.), Conducta operante: investigación y aplicaciones (pp. 455-531) (F. López, E. Galindo e E. R. Iñesta, Trads.). México: Trillas. Publicado originalmente em língua inglesa em 1966.

Baker, T. W., \& Ziegelbauer, D. (1969). Effect of CS position reversal on extinction in shuttle air-blast avoidance. Psychonomical Science, 17, 290-291.

Baron, A. (1991). Avoidance and punishment. In I. H. Iversen \& K. A. Lattal (Eds.), Techniques in the behavioral and neural sciences: Experimental analysis of behavior (Part 1, pp. 173-217). Amsterdam, The Netherlands: Elsevier.

Barry Jr., J.J., \& Degelman, J. (1961). A high-power noise amplifier with an electronic keying system. Journal of the Experimental Analysis of Behavior, 4, 85-86.

Belova, M. A., Paton, J. J., Morrison, S. E. \& Salzman, D. (2007). Expectation modulates neural responses to pleasant and aversive stimuli in primate amygdala. Neuron, 5, 970-984.

Caputa, M., Dok, K., \& Kurowicka, B. (1998). Behavioral approach to the study of the upper limit of temperature tolerance in rats. Physiology \& Behavior, 65, 183-189.

Carvalho Neto, M. B., Maestri, T. C. \& Menezes, E. S. R. (2007). O jato de ar quente como estímulo aversivo: efeitos supressivos da exposição prolongada em Rattus norvegicus. Acta Comportamentalia, 15, 171-190.

Carvalho Neto, M. B., Maestri, T. C., Tobias, G. K. S., Ribeiro, T. C., Coutinho, E. C. N. N., Miccione, M. M., Oliveira, R. C. V., Ferreira, F. S. S., Farias, D. C. \& Moreira, D. (2005). O jato de ar quente como estímulo punidor em Rattus norvegicus. Psicologia: Teoria e Pesquisa, 21, 335-339.

Catania, A. C. (1999). Aprendizagem: comportamento, linguagem e cognição. $4^{\text {a }}$ Edição (D. G. Souza ... [et 
al], Trads). Porto Alegre: Artmed. Publicado originalmente em língua inglesa em 1998.

Clark, M. G., Vasilevsky, S., \& Myers, T. M. (2003). Air and shock two-way shuttlebox avoidance in C57BL/6J and 129X1/SvJ mice. Physiology and Behavior, 78, 117-123.

Davidson Jr, R. S. (1966). Operant stimulus control applied to maze behavior: heat escape conditioning and discrimination reversal in Alligator Mississippiensis. Journal of the Experimental Analysis of Behavior, 9, 671-676.

Domjan, M., \& Burkhardt, B. (1993). The principles of learning and behaviour. Pacific Grove, CA: Brooks/Cole.

Estes, W. K. (1944). An experimental study of punishment. Psychological Monographs, 57, 1-40.

Green, K. F., Hart, G. L., \& Hagen, H. S. (1981). Toxic heat as a UCS in conditioning in internal and external milieus in rats. Physiology \& Behavior, 27, 77-82.

Harikai, N., Sugawara, T., Tomogane, K., Mizuno, K., \& Tashiro, S. (2004). Acute heat stress induces jumping escape behavior in mice. Physiology \& Behavior, 83, 373-376.

Herman, R. L., \& Azrin, N. H. (1964). Punishment by noise in an alternative response situation. Journal of the Experimental Analysis of Behavior, 7, 185-188.

Holz, W. C., \& Azrin, N. H. (1962). Recovery during punishment by intense noise. Psychological Reprints, 11, 655-657.

Knutson, J. F., \& Bailey, M. I. (1974). Free-operant escape-avoidance of noise by rats. Journal of the Experimental Analysis of Behavior, 22, 219-229.

Lerman, D. C. \& Vorndran, C. M. (2002). On the status of knowledge for using punishment: implications for treating behavior disorders. Journal of the Applied Analysis of Behavior, 35, 431-464.

Lipton, J. M., Avery, D. D., \& Marotto, D. R. (1970).
Determinants of behavioral thermoregulation against heat: Thermal intensity and skin temperature levels. Physiology \& Behavior, 5, 1083-1088.

May, R. B., Tolman, C. W. \& Schoenfeldt, M. G. (1967). Effects of pre-training exposure to the CS on conditioned suppression. Psychonomical Science, 9, 61-62.

Myers, T. M., Cohn, S. I., \& Clark, M. G. (2005). Acquisition and performance of two-way shuttlebox avoidance: Effects of aversive air intensity. Learning and Motivation, 36, 312-321.

Ploghaus, A., Tracey, I., Clare, S., Gati, J. S., Rawlins, N. P., \& Matthews, P. M. (2000). Learning about pain: the neural substrate of the prediction error for aversive events. Proceedings of the National Academy of Sciences, 97, 9281-9286.

Ray Jr., A. J. (1966). Shuttle avoidance: Rapid acquisition by rats to a pressurized air unconditioned stimulus. Psychonomical Science, 5, 29-30.

Ray Jr., A. J., \& Lenz, P. (1968). Pressurized air shuttle avoidance and emotionality. Journal of Genetic Psychology, 112, 43-48.

Riess, D. (1970). The buzzer as a primary aversive stimulus: I. Unconditioned acceleration and summation of conditioned and unconditioned acceleration. Psychonomical Science, 21, 167-169.

Rohles Jr., F. H. (1965). Wind as an aversive stimulus. Journal of the Experimental Analysis of Behavior, 8, 203-205.

Vierck Jr, C. J., Kline IV, R., \& Wiley, R. G. (2004). Comparison of operant escape and innate reflex responses to nociceptive skin temperatures produced by heat and cold stimulation of rats. Behavioral Neuroscience, 118, 627-635.

Recebido em 8 de agosto de 2009 Aceito em 17 de novembro de 2009 


\title{
Joâo Claudio Todorov
}

UNIVERSIDADE CATÓLICA DE GOIÁS E INSTITUTO DE EDUCAÇÃO SUPERIOR DE BRASÍLIA, BRASIL

\section{RESUMO}

O artigo mais citado da história de 70 anos da análise do comportamento confirma o prometido por Skinner em artigo publicado 20 anos antes e homenageia Thorndike, o precursor da tese de seleção do comportamento por suas conseqüências. Ao criticar as teorias da aprendizagem da época (1950) por falta de base empírica Skinner mostrou alguns procedimentos experimentais simples por onde se poderia começar a trabalhar com dados conceitos teóricos como decisóes e preferências. O artigo de Herrnstein de 1970 teoriza em cima de 20 anos de acumulação de dados por meio do uso desse procedimento.

Palvras-chave: Herrnstein, Skinner, esquemas concorrentes, escolhas, decisóes, preferências.

\begin{abstract}
The most cited article in 70 years of behavior analysis delivers what was promised by Skinner and honors Thorndike, the precursor of the theory of selection of behavior by its consequences. Criticizing learning theories of those times for lack of empirical basis Skinner (1950) showed some experimental procedures to collect data related to concepts like decisions and preferences. Herrnstein's article of 1970 present a theory based on 20 years of research using those procedures.
\end{abstract}

Key words: Herrnstein, Skinner, concurrent schedules, choice, decision, preference.

Quando Skinner publicou seu primeiro livro, "The Behavior of Organisms" (Skinner, 1938), Tolman, um dos grandes nomes da psicologia da aprendizagem da época escreveu-lhe uma carta elogiando o trabalho e sugerindo a introdução de uma segunda barra na câmara experimental mais tarde conhecida como caixa de Skinner. Na época Tolman estava publicando um artigo que viria a ser um clássico na literatura sobre preferências, escolhas e decisões (Tolman, 1938). Skinner demorou doze anos para seguir o conselho, mas quando o fez foi em grande estilo, em outro artigo que viria a ser clássico, sobre a necessidade de teorias da aprendizagem (Skinner, 1950/2005), um trabalho mais sobre método para o estudo experimental adequado de diversos processos de interação comportamento-ambiente do que de crítica a teorias. "Teorias da aprendizagem são necessárias?" foi seguido por um esforço monumental que envolveu anos de coleta de dados de centenas de animais, principalmente pombos, resultando na publicação do livro "Schedules of Reinforcement" (Ferster \& Skinner, 1957), obra com muitos dados e pouca teoria, um dos responsáveis pela má fama dos skinnerianos, vistos por muitos como alérgicos a teorias. Duas teses de doutorado na Universidade de Harvard estavam em desenvolvimento na época, resultando em duas publicações sobre o estudo de escolhas e preferências usando esquemas concorrentes de reforço (Findley, 1958; Herrnstein, 1961). Apesar de serem baseados

O autor é bolsista de produtividade do CNPq. Correspondência para o e-mail: joaoclaudio.todorov@gmail.com. 


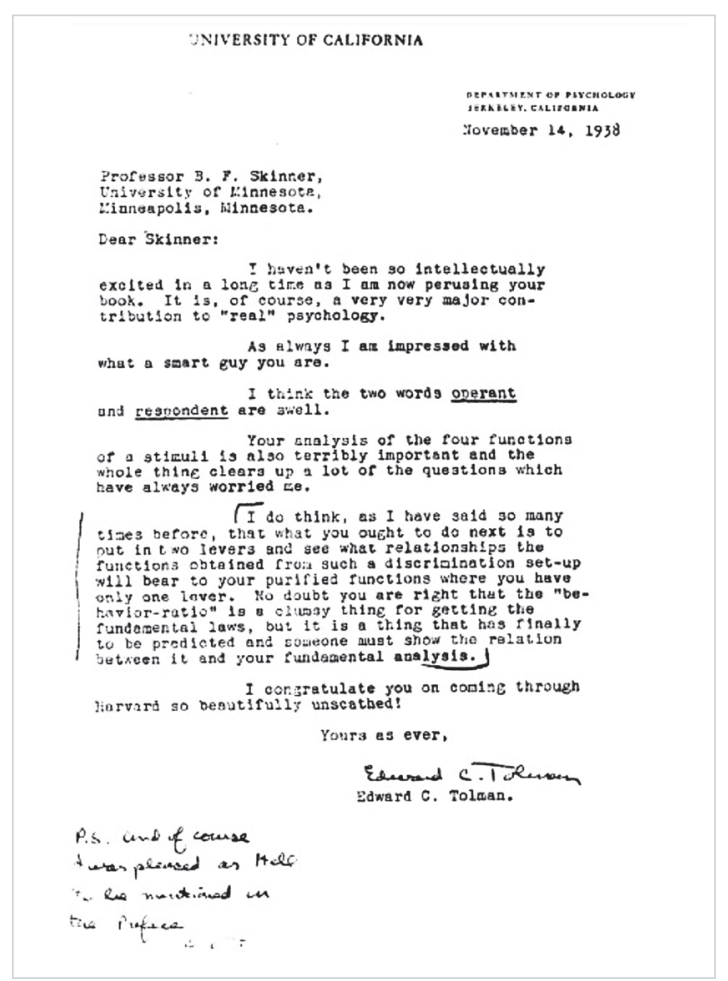

Figura 1. Carta de Tolman a Skinner comentando a publicação de The behavior of organisms. Arquivo da B. F. Skinner Foundation. Cortesia de Maria Teresa Araújo Silva.

em teses de doutorado, com pombos como sujeitos experimentais, os dois artigos mostram mais método e dados que teoria. O experimento de Findley (1958) introduz uma modificação no procedimento usado por Skinner (1950/2005), explicitando em um operandum a resposta de mudança de um esquema de reforço para outro, identificados por luzes de cores diferentes no outro operandum. Herrnstein (1961) usou o procedimento original de duas chaves de respostas e manipulou sistematicamente a distribuição de reforços programados pelos esquemas do par concorrente, obtendo relações quantitativas entre comportamento e conseqüências consistentes e uniformes.

Nos anos 60 do século passado vários experimentos com esquemas concorrentes foram publicados e vários outros, com esquemas simples ou múltiplos, também observaram rela- ções quantitativas entre freqüência de respostas e parâmetros de reforço. No artigo publicado em 1970, Herrnstein finalmente realiza a profecia de Skinner de 1950. Da sistematização de dados empíricos nasce a teoria (Todorov, 1978), resumida no que se convencionou chamar de Quantificação da Lei do Efeito: a freqüência absoluta de qualquer resposta é proporcional à sua freqüência relativa de reforço. Como corolário, tudo é escolha. Não há mais sentido em descrever apenas a relação entre uma resposta e a freqüência absoluta de reforço a ela associada sem considerar o contexto de reforço da situação. Mesmo na caixa de Skinner tradicional, com uma barra de respostas, a qualquer dado momento o rato pode responder ou deixar de responder (ocupando-se da higiene pessoal, por exemplo). Todo comportamento é escolha, e toda escolha é comportamento (Todorov, 1989/2007; Todorov \& Hanna, 2005).

"On the Law of Effect" tornou-se o artigo do Journal of the Experimental Analysis of Behavior mais citado em toda a história de 50 anos da revista. Se não for o mais importante, certamente é o que teve e tem mais impacto dentre os publicados.

\section{REFERENNCIAS}

Ferster, C. B. \& Skinner, B. F. (1957). Schedules of reinforcement. Englewood Cliffs, NJ: Prentice Hall.

Findley, J. D. (1958). Preference and switching under concurrent scheduling. Journal of the Experimental Analysis of Behavior, 1, 123-144.

Herrnstein, R. J. (1961). Relative and absolute strength of responses as a function of frequency of reinforcement. Journal of the Experimental Analysis of Behavior, 4, 267-272.

Herrnstein, R. J. (1970). On the law of effect. Journal of the Experimental Analysis of Behavior, 13, 243-266.

Skinner, B. F. (1938). The behavior of organisms. New York, NY: Appleton-Century-Crofts.

Skinner, B. F. (1950/2005). Teorias da aprendizagem 


\section{CONFIRMING SKINNER AND PRAISING THORNDIKE}

são necessárias? Revista Brasileira de Análise do Comportamento, 1 (1), 105-124

Todorov, J. C. (1989/2007). A psicologia como o estudo de interaçóes. Psicologia: Teoria e Pesquisa, 23, 57-61.

Todorov, J. C. (1978), La sistematización de datos empíricos sin (o casi sin) el auxilio de una teoría. Em P. Speller (Org.), Análisis de la conducta: trabajos de investigación en Latinoamérica. México: Trillas.
Todorov, J. C. \& Hanna, E. S. (2005). Quantificação de escolhas e preferências. Em J. Abreu-Rodrigues \& M. R. Ribeiro (Orgs.), Análise do comportamento: pesquisa, teoria e aplicação. (pp. 197-123). Porto Alegre: Artmed.

Tolman, E.C. (1938). The determiners of behavior at a choice point. Psychological Review. 45, 1-41.

Artigo convidado

Recebido em 26 de abril de 2009

Publicação referente ao 2. Semestre de 2008, impressa em maio de 2011 